\title{
Standardisierte mikroRNA-Analytik - die große Chance für Biomarkerstudien?
}

\author{
MARIA FAUTH ${ }^{1,2}$, MEIKE J. SAUL ${ }^{1}$ \\ ${ }^{1}$ FACHBEREICH BIOLOGIE, TU DARMSTADT \\ 2 PROLYTIC GMBH, FRANKFURT A. M.
}

\section{Circulating microRNAs (miRs) represent promising diagnostic and prognostic biomarkers for various diseases. Despite the high number of biomarker studies, the results of these studies are hardly repro- ducible. This makes it difficult to transfer the results into clinical appli- cation. In this context, acceptance criteria for the quantification of miRs by reverse transcription quantitative real-time polymerase chain reaction (RT-qPCR) may help to standardize miR analysis and improve reproducibility.}

DOI: $10.1007 / \mathrm{s} 12268-020-1462-7$

(c) Die Autorinnen 2020

Der therapeutische Ansatz der „personalisierten Medizin“ basiert auf der Behandlung von Patientinnen und Patienten in Abhängigkeit von genetischen Prädispositionen sowie von individuellen molekularen und zellulären Merkmalen [1]. Solche Therapieansätze stützen sich auf die Bestimmung von Biomarkern, den natürlichen Parametern biologischer Prozesse mit diagnostischer oder prognostischer Aussagekraft.

Eine individualisierte Behandlung und die flexible Überwachung des Krankheitsverlaufs werden durch ein minimal- oder nicht-invasives Verfahren, der Flüssigbiopsie ermöglicht [1]. Einer der aussichtsreichsten Biomarkerkandidaten hierfür sind extrazelluläre mikroRNAs (miRs), kleine nicht-codierende RNAs mit zentraler Rolle bei der posttranskriptionellen Kontrolle wichtiger Zellfunktionen [2]. Sie werden von verschiedenen Zelltypen z. B. direkt in die Blutbahn freigesetzt (Abb. 1). Durch ihre Kopplung an Argonaut-Proteine, Lipid-Protein-Komplexe oder ihre Verpackung in extrazelluläre Vesikel (z. B. Exosomen) sind extrazelluläre miRs vor RNase-Abbau geschützt und gelten daher als sehr stabile Biomarker [3].

Trotz der hohen Zahl von miR-Biomarkerstudien sind die meisten der beschriebenen Studien bislang schlecht reproduzierbar [4]. Die widersprüchlichen Ergebnisse sind weitgehend auf die Heterogenität der präanaly- tischen und analytischen Schritte zurückzuführen. Um valide miR-Biomarker zu entwickeln, ist daher eine Validierung und Standardisierung der Methoden notwendig [5].
Herausforderungen bei der Etablierung extrazellulärer miRs als Biomarker

Die ersten kritischen Schritte von Biomarkerstudien sind die Probenentnahme und der Probentyp (Abb. 2A). Bei der Entnahme von z. B. Blut werden verschiedene Ansätze der Blutaufarbeitung verfolgt. Der Vergleich von Studien mit unterschiedlichem Blutmaterial ist schwierig, da die miR-Konzentration durch die unterschiedliche Aufarbeitung variiert (Abb. 2B). So wird Blutserum nach der Blutgerinnung gewonnen. Während der Gerinnung kann es zur Thrombozytenaktivierung und damit zu einer unspezifischen Freisetzung von miRs kommen [6]. Dies kann zu einem verfälschten miR-Gehalt im Serum führen.

Für Blutplasma muss der Einfluss verschiedener Antikoagulantien (z. B. EDTA oder Citrat) auf die Analyse der miRs beachtet werden (Abb. 2B). Dieser Matrixeffekt kann enzymatische Reaktionen der miR-Analyse

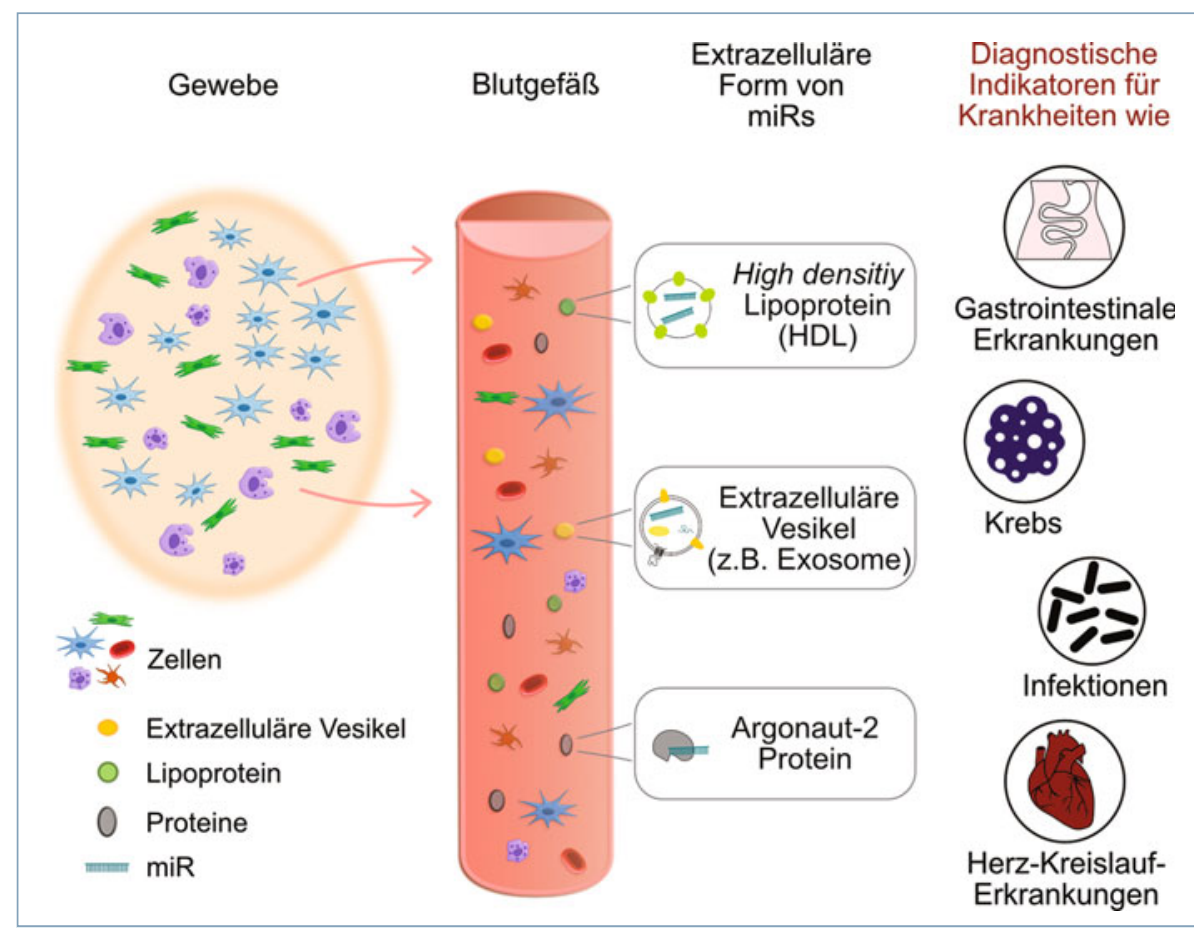

$\Delta$ Abb. 1: Extrazelluläre miRs als Biomarker. Gewebespezifische subzelluläre Komponenten im Blut, wie zirkulierende Zellen oder verschiedene Formen von extrazellulären miRs, bieten zahlreiche klinische Anwendungen für die Diagnose verschiedener Krankheiten. 
hemmen oder die Freisetzung von miRs aus Erythrozyten fördern [7]. Vor der Aufarbeitung des Plasmas beeinflussen Lagerungszeit und -temperatur den Gehalt von miRs in der Probe [8]. Zudem kann schon ein Einfrierund Auftauzyklus des Plasmas die Entstehung von schwer löslichen Proteinaggregaten fördern, in welchen miRs inkorporiert vorliegen können. Dadurch ist die Entnahme homogener Aliquote schwierig und kann die Messung verfälschen [5].

Auch die Extraktionsmethode kann zu möglicher Variabilität der Analyse führen (Abb. 2A). MiRs aus Bioflüssigkeiten werden meist mit Phenol/Guanidiniumthiocyanat (z. B. Trizol ${ }^{\mathrm{TM}}$ ) und einer Ethanolfällung oder durch Aufreinigung über silikabasierte Membranen isoliert. Abhängig von der Methode lassen sich unterschiedliche miR-Ausbeuten beobachten [5]. Dabei scheint der GC-Gehalt, die miR-Sekundärstruktur und der GesamtRNA-Gehalt einer Probe entscheidend für die Verteilung isolierter miRs zu sein [9].

Bei der Analyse intrazellulärer miRs wird eine spektralphotometrische Bestimmung der Gesamt-RNA durchgeführt, um die RNAKonzentration aller Proben für die reverse Transkription zu vereinheitlichen. Allerdings lässt sich dieses Verfahren nicht auf isolierte RNA aus Bioflüssigkeiten anwenden, da der RNA-Gehalt für diese Messung zu niedrig ist und dies zu falschen Konzentrationsmessungen führt (Abb. 2B, [8]). Daher ist eine Normierung auf konstantes Volumen von Vorteil.

Für die Identifikation von pathospezifischen miR-Expressionsmustern werden oft Hochdurchsatzmethoden (deep sequencing, microarray-Analysen) verwendet. Für die Validierung vielversprechender miR-Biomarkerkandidaten und die Übertragung in eine klinische Anwendung scheint die vergleichsweise kostengünstige RT-qPCR mit hoher Spezifität und Sensitivität am geeignetsten zu sein. Allerdings ist die RT-qPCR auch sehr sensitiv gegenüber diversen Schwankungen durch Variationen der Lyse und Aufreinigung sowie durch den Einfluss von Salzen und organischen Lösungsmitteln auf die reverse Transkription und RT-qPCR (Abb. 2B).

Solche Faktoren beeinflussen die Cq-Werte (quantification cycle) mehrfach analysierter Proben mit einer Variation von durchschnittlich zwei Prozent (CV, coefficient of variation). Diese relativ niedrigen Schwankungen verzehnfachen sich allerdings bei Bestimmung der Konzentrationen, da der Cq-Wert

\begin{tabular}{|l|l|l|l|}
\hline Probennahme & \multicolumn{2}{|c|}{ Workflow miRNA Quantifizierung } \\
\hline Probenlagerung
\end{tabular}

$\triangle$ Abb. 2: Zusammenfassung der Probleme und Lösungsansätze, die bei der Analyse zirkulierender miRs auftreten können. A, Übersicht der Quantifizierung zirkulierender miRs mittels RT-qPCR. Nach Probenentnahme kann RNA mit unterschiedlichen Methoden isoliert werden. miRs können über TaqMan ${ }^{\text {TM }}$ oder SYBR ${ }^{\circledR}$ Green-basierte RT-qPCR-Methoden quantifiziert werden. Hierbei wird die miR-Konzentration spezifisch über die Veränderung des Fluoreszenzsignals bestimmt. B, Methodische Aspekte, die bei der Analyse zu berücksichtigen sind. C, Akzeptanzkriterien als Lösungsansatz zur Sicherstellung der Reproduzierbarkeit von miR-Biomarkerstudien.

direkt proportional zum Zehnerlogarithmus der Konzentration ist. Für eine robuste Aussagekraft eigener RT-qPCR-Daten ist daher die Ermittlung der Präzision und der Reproduzierbarkeit der angewandten RNA-Isolationsmethode mit der entsprechenden RTqPCR entscheidend.

\section{Akzeptanzkriterien als erster Schritt zur Standardisierung}

Bei der Validierung wird geprüft, inwieweit die Methode bestimmte Akzeptanzkriterien erfüllt. Dazu gehört der Nachweis der Präzision und Reproduzierbarkeit der Methode für einen zuvor definierten Analyten. Wir haben dieses Prinzip auf die Analyse extrazellulärer miRs angewandt und Akzeptanzkriterien für ihre Quantifizierung in humanem Plasma durch RT-qPCR definiert (Abb. 2C, [5]). Abhängig von der untersuchten miR beobachteten wir signifikante Unterschiede in der Quantifizierung, die von Faktoren wie
Antikoagulantien, Lagerungs- oder Extraktionsmethode beeinflusst werden. Die verwendeten Methoden sollen mithilfe der definierten Akzeptanzkriterien optimiert werden, um die Standardisierung der miR-Analyse zu fördern und miR-spezifische Variationen zu vermeiden.

So sollten keine unterschiedlichen Plasmaantikoagulantien oder gar verschiedene Probentypen verwendet werden (Abb. 2C). Alternativ kann durch eine Validierung nachgewiesen werden, dass die Verwendung verschiedener Antikoagulantien oder vorhandene Proteinaggregate die miR-Analyse nicht beeinflusst [5].

Dasselbe gilt für Studien mit verschiedenen Isolationsmethoden. Die Ausbeute kann nicht nur zwischen den Methoden, sondern auch in Abhängigkeit von der zu isolierenden miR variieren. Ein Ansatz wäre hier die Gleichbehandlung von Proben und der Standards, die der Konzentrationsbestimmung 
dienen (Abb. 2C). Damit werden Unterschiede in der miR-Ausbeute bei der Berechnung der Konzentration berücksichtigt [5]. Um die RNA-Ausbeute generell zu erhöhen, werden den Proben oft zusätzliche Träger-RNAs (carrier) zugesetzt. Die Konzentration der Gesamt-RNAs in den Proben kann entscheidend für einen selektiven Verlust von miRs mit niedrigem GC-Gehalt und/oder stabiler Sekundärstruktur sein [9]. Ist die Gesamtkonzentration zu niedrig, ist oft eine geringere Ausbeute dieser miRs zu beobachten, was zu einer Veränderung der miR-Verteilung führt.

In einer Validierung werden die Präzision und Reproduzierbarkeit einer Methode mit Qualitätskontrollen (QC) bestimmt. Dabei werden den Proben miRs mit bekannter Konzentration zugefügt und in Mehrfachbestimmung isoliert und quantifiziert. Mithilfe solcher QCs kann die Präzision (CV $\leq 25 \%)$, Reproduzierbarkeit ( $\mathrm{CV} \leq 35 \%)$ und die Wiederfindungsrate (accuracy, A $\pm 30 \%$ ) bestimmt werden (Abb. 2C). Solche QCs können zur Qualitätssicherung der RT-qPCR bei jeder Messung mitgeführt werden. Bei Überschreitung der Kriterien sollte die Messung entsprechend wiederholt werden.

Für die Analyse extrazellulärer miRs wurde bislang noch keine universelle endogene Kontrolle gefunden. Daher werden den Proben zur Normierung technischer Schwankungen nicht-konservierte synthetische miRs im ersten Schritt der Extraktion zugefügt (spike in-Kontrolle). Doch auch hier sind miR-spezifische Unterschiede in der Normalisierungseffizienz verschiedener spike inKontrollen zu beobachten, sodass Schwankungen teilweise nicht normalisiert oder sogar verstärkt werden [5]. Dies limitiert die Vergleichbarkeit von miR-Biomarkerstudien mit unterschiedlichen Normalisierungs- strategien und setzt eine Validierung der Kontrollen voraus.

Diese Beispiele zeigen, dass jede Änderung im Prozess von der Blutentnahme bis RT-qPCR die Ergebnisse der miR-Analyse beeinflusst. Daher wäre eine Methodenvalidierung nach jeder Änderung im Prozess einer Biomarkerstudie ratsam. Die Formulierung von Akzeptanzkriterien für die Analyse extrazellulärer miRs ist der erste Schritt zur Standardisierung von miR-Biomarkerstudien.

\section{Literatur}

[1] Pardini B, Sabo AA, Birolo G et al. (2019) Noncoding RNAs in extracellular fluids as cancer biomarkers: The New Frontier of Liquid Biopsies. Cancers 11:1170

[2] Saul MJ, Emmerich AC, Steinhilber D et al. (2019) Regulation of eicosanoid pathways by microRNAs. Front Pharmacol 10:824

[3] Mitchell PS, Parkin RK, Kroh EM et al. (2008) Circulating microRNAs as stable blood-based markers for cancer detection. Proc Natl Acad Sci USA 105:10513-10518

[4] Witwer KW (2015) Circulating microRNA biomarker studies: pitfalls and potential solutions. Clin Chem 61:56-63 [5] Fauth M, Hegewald AB, Schmitz L et al. (2019) Validation of extracellular miRNA quantification in blood samples using RT-qPCR. FASEB Bioadv 1:481-492
[6] Wang K, Yuan Y, Cho JH et al. (2012) Comparing the microRNA spectrum between serum and plasma. PLoS One 7:e41561

[7] Makhro A, Huisjes R, Verhagen LP et al. (2016) Red cell properties after different modes of blood transportation. Front Physiol 7:288

[8] McDonald IS, Milosevic D, Reddi HV et al. (2011) Analysis of circulating microRNA: preanalytical and analytical challenges. Clin Chem 57:833-840

[9] Kim YK, Yeo J, Kim B et al. (2012) Short structured RNAs with low GC content are selectively lost during extraction from a small number of cells. Mol Cell 46:893-895

Funding Open Access funding enabled and organized by Projekt DEAL. Open Access Dieser Artikel wird unter der Creative Commons Namensnennung 4.0 International Lizenz veröffentlicht, welche die Nutzung, Vervielfältigung, Bearbeitung, Verbreitung und Wiedergabe in jeglichem Medium und Fo
erlaubt, sofern Sie den/die ursprünglichen Autor(en) und die Quelle erdaubt, sofern Sie den/die ursprünglichen Autor(en) und die Quelle
ordnungsgemäß nennen, einen Link zur Creative Commons Lizenz beifügen und angeben, ob Änderungen vorgenommen wurden. Die in diesem Artikel angeben, ob Anderungen vorgenommen wurden. Die in diesem Artikel
enthaltenen Bilder und sonstiges Drittmaterial unterliegen ebenfalls der genannten Creative Commons Lizenz, sofern sich aus der Abbildungslegende nichts anderes ergibt. Sofern das betreffende Material nicht unter der genannten Creative Commons Lizenz steht und die betreffende Handlung nich nach gesetzlichen Vorschriften erlaubt ist, ist für die oben aufgeführten Weiterverwendungen des Materials die Einwiligung des jeweiligen

Korrespondenzadresse:

Dr. Meike J. Saul

Fachbereich Biologie

Technische Universität Darmstadt

Schnittspahnstraße 10

D-64287 Darmstadt

saul@bio.tu-darmstadt.de

Maria Fauth
Jahrgang 1992. 2012 Biologiestudium (B. Sc.), Universität Gießen. 2015 Master-
studium der technischen Biologie, TU Darmstadt. Seit 2018 Promotion bei Dr. M. J.
Saul am Fachbereich Biologie, TU Darmstadt, in Kooperation mit Prolytic GmbH,
Frankfurt a. M.

